\title{
Environmentally Lean Production: The Development and Incorporation of an Environmental Impact Index into Value Stream Mapping
}

\author{
T. J. Roosen and D. J. Pons \\ Department of Mechanical Engineering, University of Canterbury, Private Bag 4800, Christchurch 8140, New Zealand \\ Correspondence should be addressed to D. J. Pons; dirk.pons@canterbury.ac.nz
}

Received 7 December 2012; Accepted 16 February 2013

Academic Editor: Gabor Szederkenyi

Copyright (C) 2013 T. J. Roosen and D. J. Pons. This is an open access article distributed under the Creative Commons Attribution License, which permits unrestricted use, distribution, and reproduction in any medium, provided the original work is properly cited.

\begin{abstract}
There is a need to include environmental waste alongside other lean wastes. Current concepts of environmental waste focus on the total production of waste from a plant. However waste is generated by individual processes within the production. Therefore focused management of waste requires engineers to know what and where waste is being generated. This is often simply not known with any accuracy. This work offer a solution by developing a method to integrate environmental waste into the lean method of Value Stream Mapping (VSM). Specifically it integrate corporate environmental standards with the VSM process, thereby permitting established lean improvement process to be focused at specific environmental improvement actions. Application of the method is demonstrated in a manufacturing setting, representing a variety of environmental impacts. The deployment is capable of being generalised to any number of environmental factors. It is able to represent a customised waste index for a particular industry. Several ways to represent the multidimensional environmental wastes were explored via industry focus group. The resulting method can be used by production staff to quantify environmental impacts at the level of the individual process and aggregated to report wastes for the whole value stream.
\end{abstract}

\section{Introduction}

Lean seeks to reduce waste in a production process. One of the more common lean management tools is the use of value stream mapping (VSM). This analyses and represents the time taken to complete a process, with a particular emphasis on time that does not add value to the product, hence nonvalue-added time. VSM is used to reduce task time and subsequently reduce company monetary overheads.

VSM focuses on "time* as a wasted consumable. However lean as a whole is concerned with many other types of waste. Consequently organisations that seek to implement lean are typically required to use different lean tools to cover the various waste dimensions of their processes. This invariably means multiple systems, with their own implementation, culture, and reporting processes. There is ongoing interest in developing integrated lean systems that avoid this duplication.
One of these areas where better integration is desirable is between the time dimension as covered by VSM and the environmental waste dimension. Environmental waste is only weakly represented in current lean thinking, which tends to simply perceive waste as merely cost of the raw materials or decrements to the productivity of the production system. However, from the environmental perspective, the type of waste is important because of the different toxicity and effect on the environment. There are also problems in getting the environmental waste considerations embedded in the production activities. Collecting data on environmental waste and its impact on the environment is the easier part. The more difficult problem to overcome is the lack of vertical integration between the organisational data on environmental waste and the processes that originally created the waste (see Figure 1). It is difficult to attribute environmental waste back to its source in the production process and consequently difficult to apply the continuous improvement methods. 


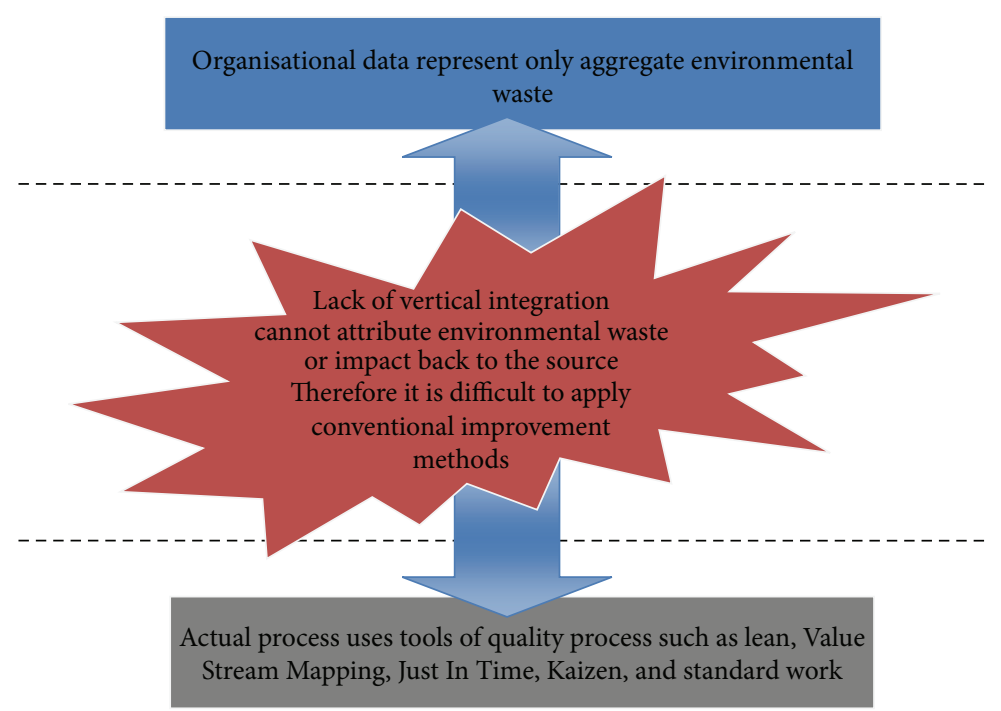

Figure 1: There is a lack of vertical integration between hard organisational data and process from which waste originated. This hinders the deployment of sustainability measures through the production system and down to the level of individual processes and operator work teams.

This paper provides a method for the integration of environmental waste into VSM processes. The particular area under examination is manufacturing, and representative data from a case are provided.

\section{Literature Review}

2.1. Lean Manufacturing and the Waste Principle. The perception of waste reduction primarily focuses on the diminution of environmental impact (ENI) through the use of traditional waste management programmes. Waste management is most often associated with objects disposed or recycled.

Lean manufacturing aims to reduce costs of production by eliminating waste and nonvalue-added activities and is a common underlying principle in many major businesses and production facilities around the world. Lean itself developed as a generalisation of the Toyota Production System (TPS), which itself was an embodiment of previous production quality systems [1-5].

In essence, lean manufacture seeks to preserve value within an organisation with overall less work and thus maximising efficiency through the reduction of waste. Though all these systems started in the manufacturing industry, the concept of "production process" can readily be applied to any other set of processes, even those that do not produce physical products. Consequently lean manufacturing has been greatly influential as a way of thinking in many industries beyond its automotive roots [6].

The TPS focused on pinpointing and eliminating waste $[2,4]$. A series of tools were developed to help map and consequently eliminate three areas. These were "Muda", also known as the seven wastes, "Muri" the overburdening of people or equipment, and "Mura" the unevenness or irregular production $[3,7,8]$. The categories developed to describe the seven primary wastes (Muda), plus the eighth waste of underutilisation of people added later in development, are shown in Figure 2.

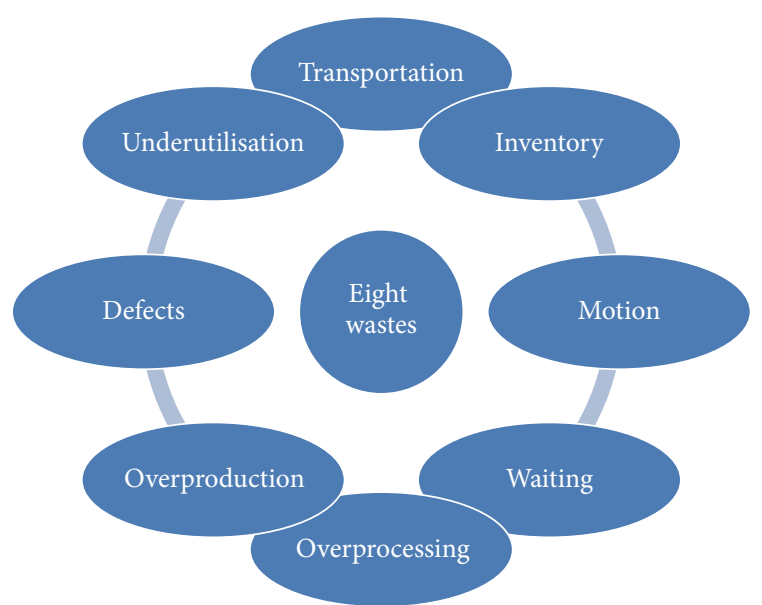

FIGURE 2: The eight wastes to be eliminated in a lean manufacturing system.

The lean methodology also subsumes many of the ideas from total quality systems, particularly the problem-solving approach. This may be summarised as investigating a problem $>$ identification of the impediments $>$ application of an improvement process $>$ ongoing cycles of continuous improvement. The concept of empowerment of operators to make suggestions and arrange their own work is also common. Indeed both quality and lean are reliant on a culture that welcomes operator engagement in the production processes beyond merely the provision of labour. The burst of activity that creates the incremental improvement is a Kaizen activity. (Kaizen refers to the lean philosophy that no process can ever be perfect, so operations must be improved continuously through waste elimination events.) In this context the term means that adequate is never good enough and that no process can ever be thought to be perfect, so therefore each process must be continually evolved and improved.

The lean production paradigm can be accomplished by applying a wide variety of lean manufacturing tools such 
as Heijunka, Six Sigma, Kanbans, First In-First Out (FIFO), Value Stream Mapping (VSM), Takt (from Taktzeit meaning cycle) time, Just In Time (JIT), Single Minute Dye Exchange (SMDE), and $5 \mathrm{~S}$ principles [4].

There have been many attempts to explore the effectiveness of different techniques used to implement lean thinking in a real practice along with examining why some techniques might be preferential to others [9]. The tools themselves are a vital component of lean implementation along with the defining culture of lean.

2.2. Value Stream Mapping. VSM is a functional method or visual flow chart by which the production process can be represented as a set of processes connected in time. The method excels at showing the time dimension, particularly the nonvalue-added or waste time. It is therefore the lean method of choice for industries where costs are mostly determined by time or where a shorter production cycle confers competitive advantage. VSM can map an entire process, supply chain network, or the subtasks within a single process. It therefore readily scales hierarchically. In addition it maps both the material flow and the information that controls production [10]. The method, being a type of flow chart, is typically implemented using a set of standard icons for information and material flow [3,11-13].

A given value stream includes all activities that contributed to a product, that is, value adding, nonvalue adding and supporting activities that are required to render the service [14-16]. The concept of waste within a manufacturing or information system can be further expanded through a categorisation of nonvalue adding (NVA) work, necessary but nonvalue adding (NNVA) work, and finally value-added (VA) work $[3,17]$. Using these principles, the baseline processes within the value stream can be established and categorised. Once the value stream has been mapped, it becomes the baseline for improvement which then can be used to help create a future state map, which represents the desired future state including process improvements and reduction of NVA and NVA waste.

VSM is widely recognised in many different organisations irrespective of the type of system under examination. Research has mostly been focused on push/pull, Kanbans, inventory control, and mixed model assembly implementation. There has been less research into adapting concepts such as JIT, continuous improvement, cycle time reduction, visual management, automation, and floor space reduction into VSM simulation [18]. Another commonly recognised flaw in VSM is the inability to map value streams other than cycle time or cost. A limited number of modified VSM concepts have been developed to cope with complex value streams primarily network value mapping and critical path Value Stream Mapping.

2.2.1. Strengths of VSM. Some of the primary strengths of VSM are $[9,18]$.

(i) VSMs are able to easily identify waste (time and cost) from the values stream; (ii) VSMs allow organisations to guide and visualise future information and material flow with iterative process improvements;

(iii) They map more than just waste and allow source and root cause to be examined,

(iv) They provide simple and objective analysis of complex systems.

2.2.2. Limitations of VSM. As with all processes, VSM has associated weaknesses inherent within the system design that limits the ability of VSM to be applied in every circumstance. A variety of limitations inherent in VSM are described below $[9,16,18,19]$.

(i) Static tool that captures snapshot-in-time not continuous flow,

(ii) Future state assumes every Kaizen will be fully completed,

(iii) Editing VSMs drawn by hand is cumbersome,

(iv) Detail capture of value stream is limited, especially in more complex multi stream systems,

(v) VSM does not represent spatial layout and consequent impacts of distance.

2.3. Methods of Environmental Waste Management. Waste management is the processing, collection, and transportation of waste (defined as "non-wanted things, that are perceived to have no purpose or value") to recover residual value or reduce consequences for the natural environment. Arguably the most widely used and universal waste management focused system is the ISO 14000 which is a family of standards relating to environmental waste management [20,21]. They assist an organisation in minimising how their operations or processes can negatively affect the environment (i.e., cause adverse changes to air, water, or land). The ISO 14031 standard (from the ISO family) relates to Environmental Performance Evaluation (EPE) and is a management system which aims to assist organisations in identifying their environmental impacts by determining which aspects will be treated as significant, setting criteria for environmental performance, and assessing their environmental performance against these criteria. As part of the IS0 14000 family, another approach is found within the ISO 14040 set of standards, described as the Environmental Management-Life Cycle AssessmentPrinciples and Framework. The principal definition of the "Life Cycle Assessment" (LCA) is the assemblage and evaluation of the inputs, outputs, and potential environmental impacts of a product system throughout the product's life cycle. The LCA model is a more focused approach to waste management than ISO 14031.

Cradle to Cradle (C2C) is a methodology that uses biomimicry to compare and analyse the human resource system as a biological organism where materials and resources are modelled as nutrients in a health metabolism. The initial coining of the term was by Walter R. Stahel in the 1970s, but it was not until a modification of the Life Cycle Assessment saw the birth of the $\mathrm{C} 2 \mathrm{C}$ ideology through the publication 
of Cradle to Cradle: Remaking the Way We Make Things [22]. The primary theory of the $\mathrm{C} 2 \mathrm{C}$ principle is the idea of regenerative design in which every product is produced in a way in which it ensures recyclability of the resource.

The polluter pays principle (PPP), also known as extended producer responsibility (EPR), emphasises that the summation of all environmental costs throughout the life cycle of any product should be reflected in the market price of that product. PPP aims to change the waste paradigm from a governmental focus on waste and environmental initiatives to corporate or manufacturing entities which produce the waste and thus should also deal with waste impacts and disposal. This would mean that manufacturers would absorb greater responsibility in the cleaning, storing, recycling, and reuse of waste produced. This type of thinking has increasingly affected national policy formulation. Therefore it is becoming increasingly important for manufacturers to develop systems to better manage their environmental waste. The preferential method of waste management would be prevention and minimisation of waste at point-of-generation, as opposed to disposal and energy recovery. Hence it is desirable to include environmental waste into lean thinking.

2.3.1. Waste Management Indices. Once an overall waste management framework is determined, it is crucial to then decide on an appropriate index in which specific environmental performance factors can be evaluated against. There exist several methods in which the environmental consequences can be measured or evaluated directly. It should be noted that a majority of the indices do not directly account for the principles of a Lean Manufacturing Programme. The ISO 14031 standards highlight the development of specific metrics through indicators. The process of choosing the indicator may include choosing from existing indicators or developing new indicators. (This standard describes the two general categories for Environmental Performance Evaluation (EPE) as Environmental Performance Indicators (EPI) or Environmental Condition Indicators (ECI). EPI can be further broken down into Management Performance Indicators (MPI) and Operation Performance Indicators (OPI). MPI is a type of EPI that provide information about management's efforts to influence the overall environmental performance of the organisation. On the other hand, OPI provides information about the environmental performance of an organisation's operations. Examples of how these three indicators interrelate are given in ISO 14031:2000.)

The US Environmental Protection Agency [20] environmental toolkit provides assistance in developing an environmentally conscious organisation. The most relevant features of the EPA toolkit relate to identification of environmental wastes and Environmental Value Stream Mapping (EVSM) adaptation. This discussion is primarily interested in the identification of wastes. Initially the toolkit describes links between the "seven wastes" and environmental wastes in identifying critical ENI. The EPA toolkit further explores the ability of targeting environmental waste in an organisation.

Environmental Management Accounting (EMA) (As described by United Nations Division for Sustainable Development UNDSD 2001) is a combined process that provides a method to translate data from financial accounting, cost accounting, and mass balance to improve material efficiency and reduce environmental impacts [23]. The primary focus of EMA is an assessment of the total annual environmental expenditure on emissions' treatment, disposal, and environmental protection and management. In essence EMA sets up procedures for internal decision making which include both physical procedures for material and energy consumption, flows, and final disposal and monetarized procedures for costs, savings, and revenues related to activities with a potential ENI. The total emissions method seeks to determine (through empirical analysis) evidence of a link between lean production practices and environmental performance [24]. The method explores three interrelated hypotheses. The hypotheses state that the more an organisation establishes lean principles, the more likely it will adopt formal environmental management systems, the less likely it will generate waste, and finally, the lower its emissions will be. In other words, an organisation's environmental performance could be defined by the degree it emits toxic pollutants [25]. The systematic (or strategic) environmental assessment (SEA) incorporates environmental considerations into policies, plans, programmes, and strategies of an organisation [26, 27]. Life Cycle Assessment (LCA) is a core concept in the development of environmentally conscious design and cleaner practices in industry and involves the evaluation of environmental burdens associated with product, process, service, or practice. Volvo along with the Federation of Swedish Industries jointly developed an Environmental Priorities Strategies (EPS) system to select appropriate materials to use during construction of its products $[28,29]$. This method is based on environmental indices calculated for specific materials.

Another possible cumulative measurement for wastes is the use of a "carbon footprint" analysis in which waste of a very specific form can be aggregated and measured. The "carbon footprint" analysis is a method in which the total emissions of greenhouse gasses (GHG) are estimated in terms of the carbon equivalence $\left(\mathrm{tCO}_{2} \mathrm{e}\right.$-tonnes of carbon dioxide equivalent or grams of $\mathrm{CO}_{2}$ equivalent per kilowatt hour of generation $\left(\mathrm{gCO}_{2} \mathrm{eq} / \mathrm{kWh}\right)$ ) from a specific product. The measurement is taken across a product's life cycle from raw materials used in manufacturing to the disposal of the final product. Its purpose is to measure the individual gas emissions from each activity within a supply chain process and framework and attribute these to each output product [30]. A carbon footprint, in other words, is a measure of the total amount of greenhouse gas (GHG) emissions. Carbon dioxide, methane, nitrous oxide, hydrofluorocarbons, perfluorocarbons, sulphur hexafluoride, and ozone are examples. These GHG emissions are either directly or indirectly caused by an activity or are accumulated over the life stages of a product. Toxicity was another possible measure of environmental impact, particularly the impact of a set process with respect to human health. Initial investigation of the use of toxicity as a potential EIF, particularly $\mathrm{LD}_{50}$, was discarded due to the high degree in variability of data available for any substance measured. High use of estimated data along with large uncertainties and safety factors did not promote 
the use of this particular EIF as a contribution to the total Environmental Impact Index (EII).

The Global Report Initiative [31] promotes economic, environmental, and social sustainability. GRI provides companies and organisations with a sustainability reporting framework. The framework includes identification of a variety of aspects oriented towards long-term sustainability for the often described economic, environmental, and social categories. Within the environmental dimension is a section with a number of aspects concerning emissions, effluents, with both core and additional performance. Other performance indicators of the GRI (environment) include the aspects of materials, energy, water, and biodiversity making a total of 30 performance indicators. The GRI has become a widely used methodology for companies to measure and report on their sustainability practices with specific measurements identified.

\section{Purpose: A Need to Integrate Environmental Factors with Lean}

Current concepts of environmental waste focus on the total production of waste from a plant. They are interested in quantifying the amount of waste and its consequences on the natural environment. Hence there is an emphasis on containing the waste within the plan boundary then applying a postproduction process to neutralise the environmental impact, and finally releasing it across the plant boundary into the environment.

There is a growing awareness of the importance of incorporating environmental factors into lean methods. There have been a number of initiatives in this direction. One was to use the Integrated Definition for Function Modelling (IDEF0) as a modelling notation to incorporate an existing waste index [32]. That work at least showed that it was conceptually possible but did not implement environmental factors into operational practices in the real industrial setting. The United States Environmental Protection Agency (EPA) has developed an environmental value stream map (EVSM) method which examines natural resource flow by expanding the mapping process to include environmental waste streams [20]. This method has been applied to reduce water consumption in an alcohol and sugar industry case study [33]. This method easily focuses on one particular form of waste but lacks the ability to focus on environmental waste as a whole or even multiple environmental waste streams.

However, clean environmental identification practices will also require reduction of waste at its point-of-generation. Waste is not generated by a plant but by individual processes within the production. Therefore focussed management of environmental waste requires that production engineers first know what the waste is and where it is being generated. This is the crux of the problem, because this is often simply not known with any accuracy. In addition, production plants are controlled and improved by lean methods, and if some waste is not visible to the lean methods, then it will not be included in the continuous improvement cycles. It is therefore imperative to identify and embed the environmental issues into the lean tools.
There have been only minor developments in creating an overall value stream environmental index and an encompassing methodology. What is needed is a way to include environmental waste alongside the other lean wastes. If this can be achieved, then the organisational momentum and culture that sustain the lean initiatives will automatically ensure that environmental waste is included in the decision making.

\section{Approach Taken}

This project was contextualised in a research collaboration with a local industry partner. This firm provides remanufacture services for a high-value precision engineering product. The firm already had an established process for implementing VSM. What was missing was the incorporation of the environmental impact of each process. This was important for the firm for two major reasons: first, that the processes can involve toxic materials and secondly that the reduction of environmental waste was seen as a strategic competitive advantage.

We approached this problem in the following way. First, we created a composite environmental waste index. We used a variety of environmental impact factors, which were then integrated to form a single new impact index that was relevant to the operational purpose of the firm. We created several different concepts for how such an index might be visually represented within the VSM framework.

Second, we tested the relevance of these concepts within the firm. Focus groups within the industry were used to identify the waste types and index factors that were most applicable to the situation. They also selected, from among the multiple concepts, which visual representation was the best for them. The focus group was comprised of several people with a variety of roles within the firm, including engineering managers, Environment Health and Safety officers, and quality control engineers. This part of the method ensures that the results are relevant to the industrial perspective and provides a degree of confidence in the applicability. We did this with awareness that adoption within an organisational culture is important for the success of any new initiative, hence the special care to engage stakeholders in the design process.

Third, from the results of the focus group we then designed the details of an integrated environmental wasteVSM (EW-VSM) method. We shaped this around VSM as that is the dominant lean tool used in this type of industry. We found a way to represent multiple dimensions of environmental waste (in this case five) for each process in the value stream. We also found a way to represent the aggregated environmental waste for the whole value stream. This permits the methodology to scale with the production hierarchy.

The fourth part of our method was to deploy this EWVSM in the firm, on actual production lines. An environmental value stream map was conducted on a process that was identified to incorporate a large amount of environmental impacts such as high energy use, carbon footprint, high cost of waste removal, and toxic materials. A current state map of the process was constructed by a team including a quality engineer, VSM specialist, production workers, and 
technical manager. This exercise was conducted over a threeday period. The implementation began with a tutorial of how the environmental impact analysis methodology worked and how it was integrated with VSM use. The selected practitioners were informed of the new methodology through the use of standard operating procedures (SOP) that had been specially written.

After informing the users, the index implementation and evaluation began. The evaluation started with a review of a particular process (Annulus Filler). Once all participants were informed of the overall approach of the environmental index method and its relationship to VSM, the first stage of the analysis was instigated. The data acquisition begins with setting the initial percent target waste reduction (in this application $80 \%$ was chosen) followed by the capture of all five impact factor components. The data capture included calculation of all carbon footprint data by hand, perceived impact, determining cost to remove waste, volume of waste removed and remaining, and finally the site based Risk Register values for each process. The environmental impact factors were then aggregated into the single environmental impact index for each of the nine stages of the VSM. The VSM with added environmental impact index data bar and summary system radar chart was then analysed along with the process radar charts to determine which process had the highest environmental impact. Finally, after all information was captured as required, the environmental value stream ladder was added to the VSM, as well as Kaizen events identified.

The fifth and final part of our approach was to survey users for their responses to the method. We did this by a survey. We were interested in the relevance and ease-of-use from the perspective of industry practitioners. This part of the method was therefore a check on the applicability of the EW-VSM construct. The survey questions are included in the results. The respondents were from those who had participated in the EW-VSM as well as other roles within the plant. Ethics approval was obtained for the survey from the University of Canterbury.

\section{Results}

5.1. Environmental Impact Index (EII). Several factors relating to the use of an index at the local industry based sponsor were required to be taken into consideration when developing the appropriate aggregated composite EII scale. The first key factor for aggregated scales is the need for an index that can consider the broader definitions of waste and environmental impact and accommodate the specific operational characteristics and strategic purposes of the organisation. A design with a multileveled weighting scale can accommodate a wide variety of EIF.

A series of nine possible environmental waste impact indices were initially examined. The EPA toolkit, EMA method, Emissions index, SEA index, and ISO 14000 have all been omitted at this stage of the project due to several limitations. Of these, the EPA toolkit, EMA method, and ISO 14000 were eliminated due to their low scores for ease of use, ease of integration, and adaptability. The Emissions index and
SEA index suffer from being too specific and inflexible in accommodating different forms of waste or environmental impact scenarios.

The surviving candidate indices were an adapted Volvo environmental priority system, simple carbon footprint index, GRI index, simplified risk and consequence index, and a custom scale. Benefits of these indices include the following:

(i) ability for some indices to accommodate multiple environmental factors (custom scale),

(ii) some proposed indices are widely used and recognized (GRI and ISO),

(iii) ability to adapt the index is recognized as a key benefit (custom scale),

(iv) ability to quickly and effectively reflect poor performing processes,

(v) ability for practitioners of various skill levels to use and operate.

Detriments of these selected indices include:

(i) some indices are based on single environmental factors (Volvo and carbon footprint),

(ii) some indices (including custom scale) are not recognized or officially vetted,

(iii) overly complex index creation (GRI and ISO).

5.2. Conceptual Design of an Index for Environmental Waste. We applied a conceptual design process to the development of the Environmental Impact Index (EII) and its visual representation. We did this because representation is an important factor in usability, and we were specifically interested in a scale design that would be easy to implement. Thus, we were also designing for change management. For this reason the process of design specifically included focus groups from within the industry under examination.

The study examined possible visual displays to represent the chosen index. We also needed the representation to be easily integrated into current VSM maps. To consolidate the disparity gap between overall site waste data and process level information, two main design criteria were required to be met. The first element required to consolidate the disparity was to create or modify an appropriate waste index and encompass this index into an overall evaluation methodology that could be used to determine specific environmental impacts at the process level. The second criteria required to be fulfilled was to create a robust visual representation method that would effectively highlight high environmental impact processes that required Kaizen (waste reduction) to initiate.

Several concepts were explored through focus group review sessions. These concepts included a bar graph display, representative symbols, and simple process flow charts, as shown in Figure 3. Participants selected a coloured flow process chart, for clarity of communication and ease of integration with VSMs. The summary of the EII may then be displayed as an environmental waste impact ladder below the current lead time ladder as shown in Figure 3. 


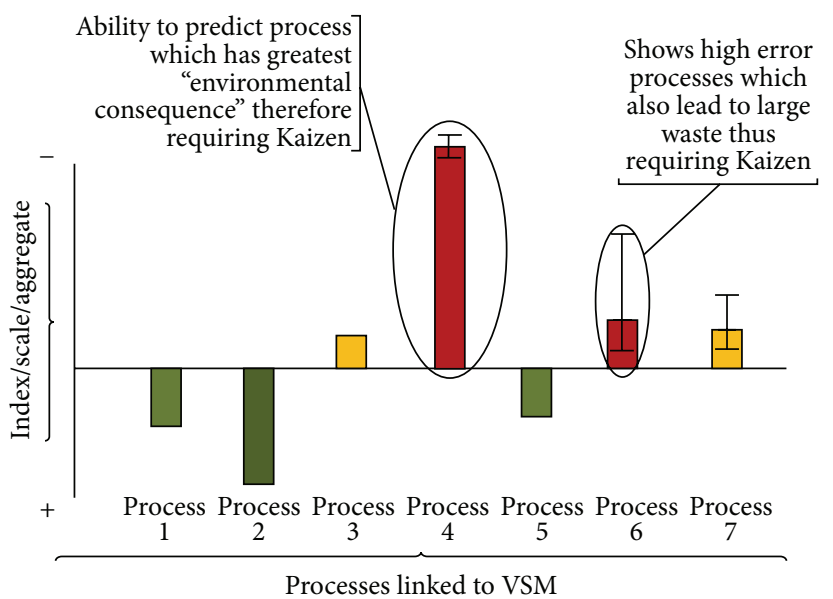

(a) Bar graph concept

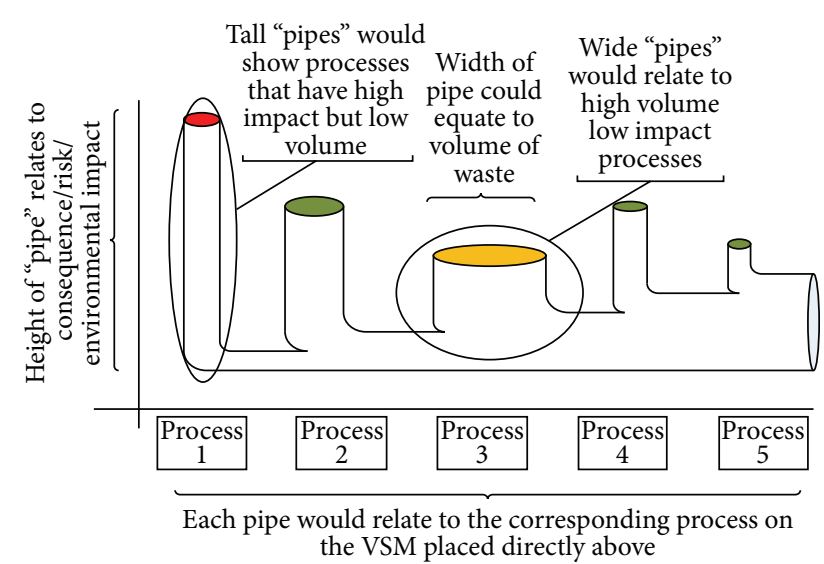

(b) Waste pipe concept

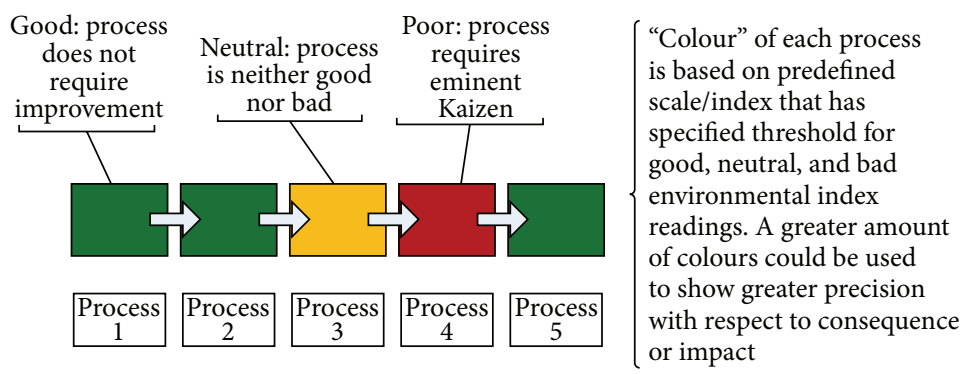

Rainbow chart display placed directly below VSM relates to each individual process

(c) Process chart concept
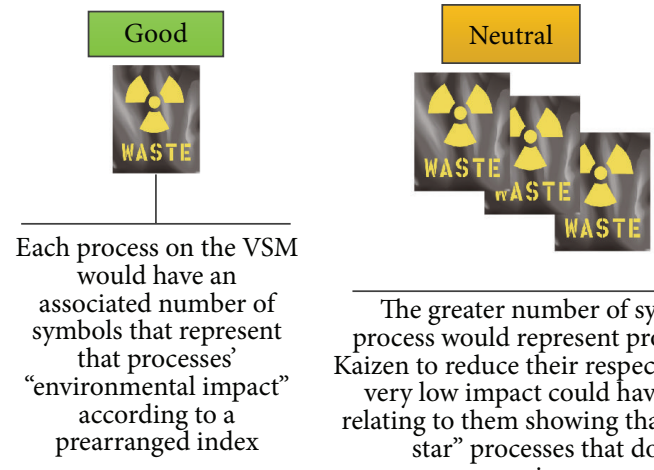

The greater number of symbols associated with each process would represent processes that immediately need
Kaizen to reduce their respective impact. Processes that have Kaizen to reduce their respective impact. Processes that have
very low impact could have a series of positive symbols relating to them showing that they are "top quality" or "
star" processes that do not comparatively need improvement/reduction

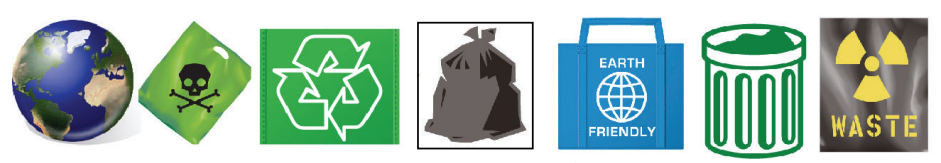

Use of different symbols can evoke a great emotional response. Also the use of positive imaging such as an earth or recycle symbol could encourage waste reduction

(d) Symbolic representation concept

Figure 3: Summary of initial visual display concepts.

Following further industrial practitioner based focusgroup review sessions with leaders in the Environment Health and Safety (EH and S), lean, and VSM (Value Stream Mapping) groups, a final customised index was chosen which incorporated various aspects of the previously described standards and indices. The most favourable index by general consensus was a customizable index that would allow the organisation to modify the index based on current site objectives and organisational purpose. A custom scale was also deemed the most preferred option because it allowed 
a balance to be created between accuracy of results, adjustability of index, and adaptability of the applied method to highlight high environmental waste impact process. An important specification identified by the focus group was to develop a composite index to be customisable to allow for future modifications as a result of changes to the organisational purpose of site goals, essentially future proofing of the methodology and index. Five EIF were chosen, reflecting the current strategic goals and organisational purpose of the particular industrial application. Descriptions of the chosen set of EIF for this application were as follows:

(i) Carbon footprint.

(ii) Perceived impact of waste (levels 1-10):

(a) Level 1: relates to near zero or minimal perceived human impact such as paper or storm.

(b) Level 5: relates to medium level of perceived human impact such as sewage.

(c) Level 10: relates to very high perceived human impact such as anthrax, radiation, or asbestos.

(iii) Cost of cleanup/remediation per $\mathrm{kg}$.

(iv) Removed waste volume $\times$ Site Environmental Risk Register value (based on ISO 14001 standards),

(v) Remaining waste volume $\times$ Site Environmental Risk Register value (based on ISO 14001 standards),

These cover all the factors that the focus group deemed pertinent to the site. However we note that the method is able to accommodate different factors and different numbers thereof, and we recommend that practitioners give thought to the wastes appropriate in their own situation rather than unthinkingly adopting the above list.

\subsection{Creation of Composite Index for Environmental Waste.} Creating a composite index consists of several key stages, the initial EIF estimation, determination of an average EIF, and aggregation of the final EII. This overall process is shown in Figure 7 . The aggregation of the composite index starts with the definition of the chosen environmental impact factors (EIF) shown in Figure 4. These interchangeable factors are the foundation for which the final EII will be based on and must be selected carefully to reflect the organisational purpose, goals, and environmental aims of the organisation in question. The chosen factors used in this particular application were decided through a series of focus group discussion as discussed previously.

The second aspect that must be defined is the scaling factor (SF). This element allows a layered system approach to be undertaken when determining which EIF is most important from a customer, practitioner, or manufacturing perspective. This preweighting also allows compensations to be made for low numerical valued EIF. At the outset, the SF would remain one unless a specific EIF needs to be highlighted or targeted. If a larger SF is required the practitioners have been advised to increase the SF in increments of 10 until a suitable value is reached, reducing the complexity of determining an appropriate number. This SF is used as an alignment modification factor to reduce or enlarge the importance of any of the chosen EIF. This might be useful to reflect a changing organisational strategic purpose, for example placing greater importance on, say, carbon footprint. By increasing the SF of the carbon footprint aspect, the company would effectively increase the percent contribution of that EIF to the overall index. Importantly the production improvement processes inherent in the lean systems will automatically refocus to reduce this particular waste.

Once the appropriate EIF have been confirmed, the data collection for each EIF begins. To compensate for inaccurate, limited, or estimated data collection of EIF, a project evaluation and review technique (PERT) analysis was used to determine an average EIF value. This proceeds from fitting a beta probability distribution to three estimates, shown in (1) and Figure 5. The EIF values are separated into Pessimistic $(P)$, Expected $(E)$, and Optimistic $(O)$ values. The distribution is weighted towards the expected EIF value, as per the function for the mean of the beta distribution, and this also minimises extreme data outliers such as an overly optimistic or pessimistic evaluation:

$$
\text { EIF estimated }=\frac{(O+4 E+P)}{6} .
$$

After the mean EIF value is determined, the EIF is then multiplied by a scaling factor (SF), as determined above. The next stage of the aggregated composite index is to assimilate the various EIF into a single index. This is determined by adding the vector magnitude of each EIF together, as shown in (2):

$$
\begin{aligned}
& \text { EII (vector magnitude) } \\
& \qquad \sqrt{\left(\mathrm{EIF}_{1} * \mathrm{SF}_{1}\right)+\left(\mathrm{EIF}_{2} * \mathrm{SF}_{2}\right)+\cdots+\left(\mathrm{EIF}_{n} * \mathrm{SF}_{n}\right)}
\end{aligned}
$$

There are several reasons for using a vector magnitude to determine the final EII. The first is that this permits any number of waste dimensions to be consolidated to a single value; that is, it makes the method scalable. A representation of a 3-dimensional waste problem is shown in Figure 6, and although a graphic representation is unavailable for the general $n$th dimensional problem, the vector magnitude still works. (This reason relates to the theoretical modelling used to address the problem and create a suitable solution. The approach was used to examine if the application of risk maps and consequence scales, representing environmental risk, could be used to provide a single valued solution. This concept of a risk map was replaced by a model in which the $x$ and $y$ axes described EIF characteristic of carbon footprint and volume of waste for a specific process. This model was further expanded to include a greater number of axes that represented different appropriate EIF. The end result was the creation of an $n$th dimensional model that could be used to describe any number of EIF. Finally a five-dimensional model was chosen, with each EIF being represented by a separate axis. Each process could then be mapped in accordance to the contribution of EIF, represented by a separate axis. This 


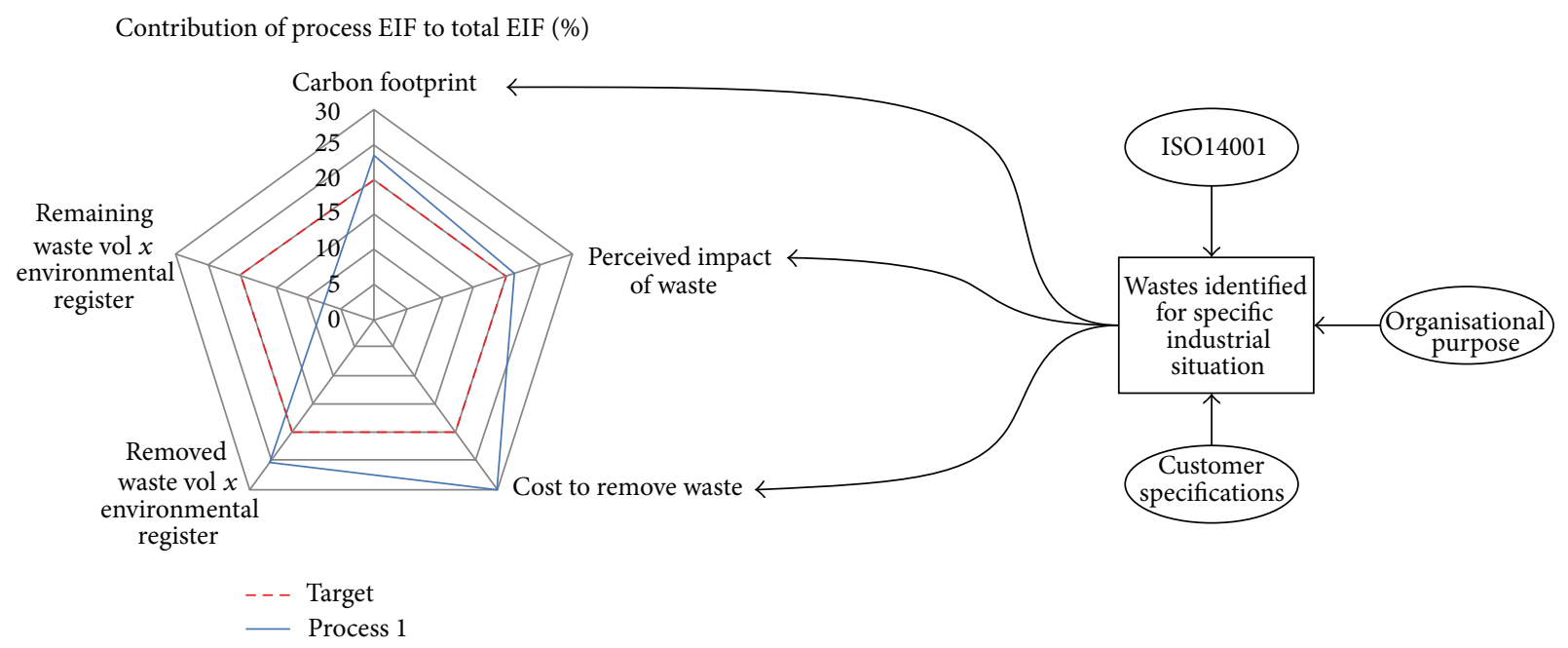

FIGURE 4: Initial environment impact factors consideration and subsequent radar chart for each process in value stream.

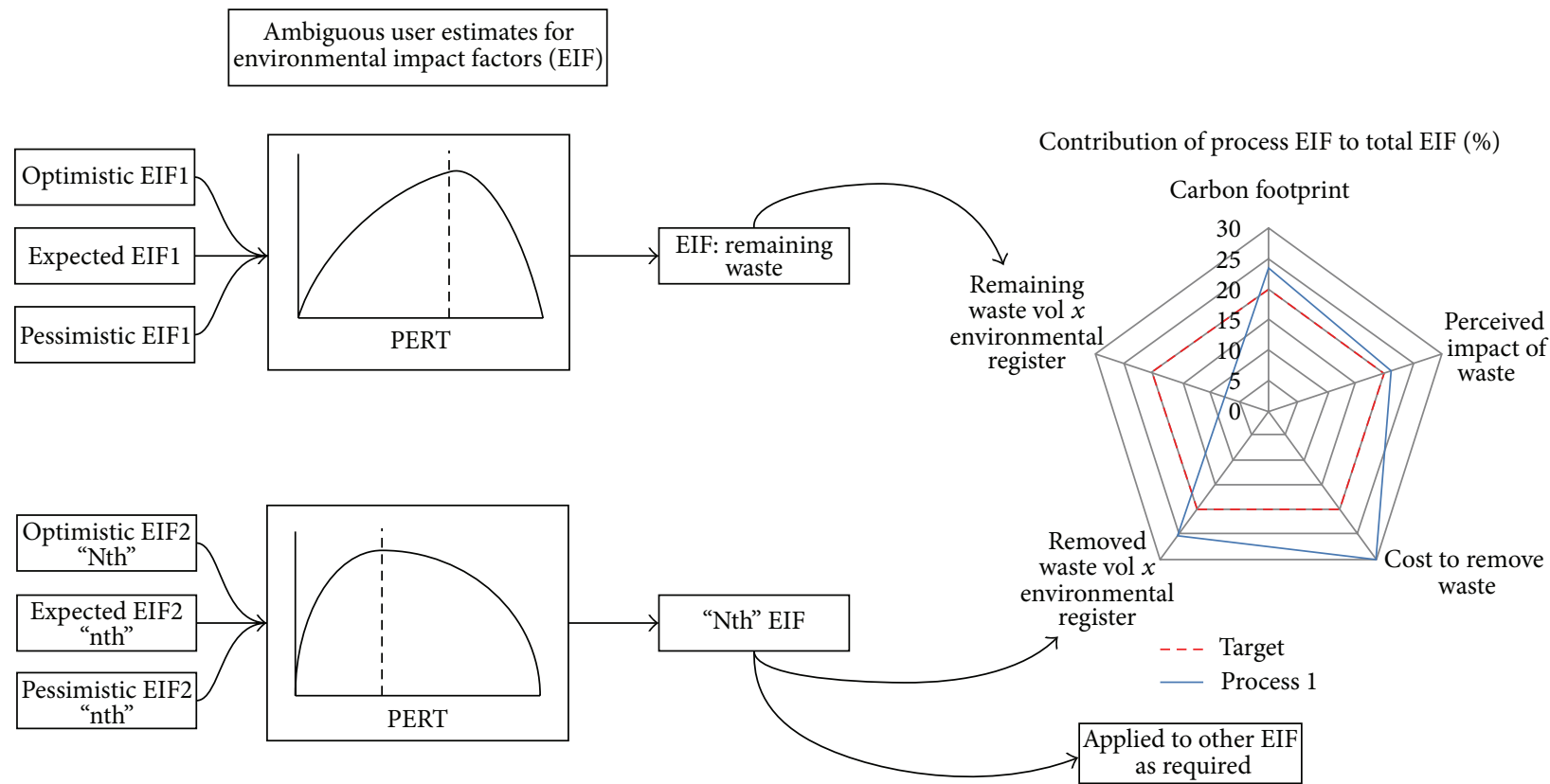

FIGURE 5: Method of determination for each environmental impact factor value using the PERT three-point estimation.

resulted in a representative 5-dimensional vector for each process, shown by a simplified illustration in Figure 6. The vectors describing each process could then be consolidated into a single valued unit through the use of the vector magnitude equation. This also means that with the addition of any extra "dimensions" describing a different EIF, final solution can be easily adjusted by adding in another vector component.)

The second reason for using a vector magnitude relates to the inability to simply multiply or add the EIF together. Direct multiplication or addition of the chosen EIF is not recommended as this could often result in large number valued solutions for specific processes as a result of one particularly high EIF that could skew the results. This problem is solved by using the vector magnitude equation as well as incorporating a scaling factor in the magnitude equation to ensure no single EIF or process dominates the overall analysis. Thirdly the vector magnitude approach allows for the likely event of a specific process having a zero valued EIF. If multiplication was used then the final value representing a process with a zero valued EIF would be reduced to zero, reflecting inaccurate result. The vector approach allows for any number of EIF to be zero values and still results in a final indicative EII.

Finally addition of EIF was considered a possible aggregation method but due to both large number dominance of some EIF compared to others and unit mismatch, this was discarded in favour of the vector approach. The methodology created is able to accommodate any number of types of waste, as discussed above, and we refer to this as an $n$th dimensional concept. The current model uses 5 waste dimensions. Each of 


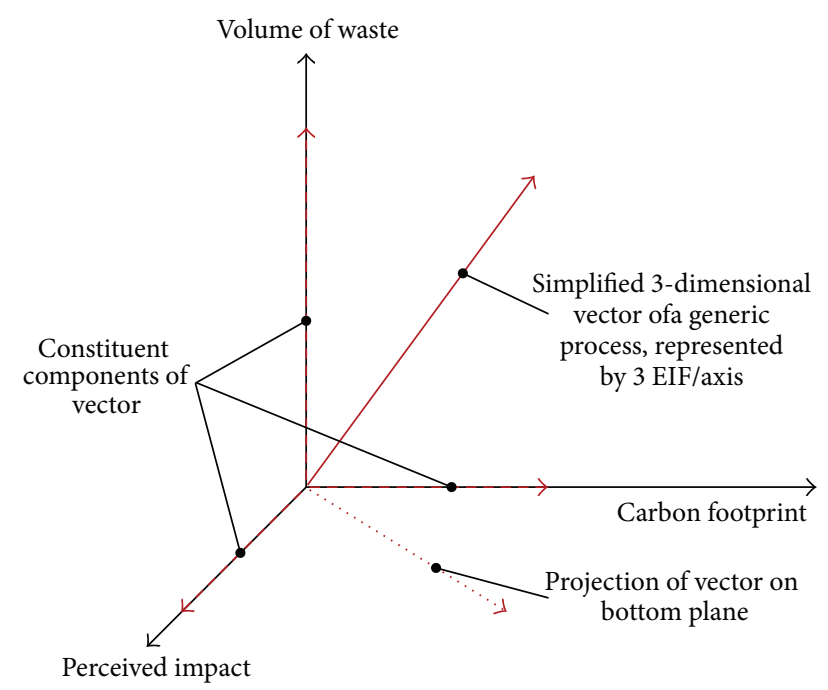

FIGURE 6: Simplified three-dimensional vector representation of generic system process.

these is represented on one axis, and additional axes may be added and further wastes are included. The vector magnitude then reduces the $n$th dimensional representation to a single value. This is useful for the ability to report summary data to managers and corporate staff and hence indicates how well the plant is meeting the strategic objectives. Thus the method integrates well with strategic management initiatives at the one level and lean improvement (via VSM specifically) at the operational level.

5.4. Identifying Environmental Kaizen Opportunities for Improvement. The purpose of lean initiatives is to identify areas for continuous improvement. These improvement foci are termed Kaizen events. Also important in lean is the realisation that not everything can be improved, because of finite resources, and it is therefore important to be able to identify which deficiencies are most worth targeting. In the case of value stream mapping, it is usual to use a burst symbol to represent the Kaizen events on the VSM chart. Also, VSM uses the concept of future state to identify the target reduction in nonvalue adding times. In the case of the environmental VSM approach described here, the Kaizen concept is directly applicable. It is straightforward to identify where to apply the environmental Kaizen, based on the process activities with the highest waste scores. Contextual knowledge of the plant may then be used to further identify which processes are likely to be more or less amenable to change. Note that the environmental Kaizen are not necessarily at the same location as those for the standard VSM. This is because the one set of process improvements are focussed on the environmental issues and the other on the temporal. (We use a green burst symbol to show the environmental Kaizen and yellow for the temporal.)

In application, the selected environmental impacts are integrated into a single EII, and a series of radar charts are created. These display the performance of individual processes and the overall system. Radar charts and conditional formatting are then used to identify the processes which required environmental Kaizen initiatives. The first set of radar charts used are at the process level and break down each individual processes performance compared to an overall threshold value (see Figure 7). This threshold may be determined by creating an overall "target" percent based value of the maximum calculated index. The highest index would be multiplied by the high and low percent targets. These percents are then used across the entire system to determine good, neutral, and bad performing processes.

The radar charts are used in two ways. The first radar chart ((B) in Figure 7) is a summary figure which displays overall process performance of each process compared to the percent thresholds. The high percent bound is determined by reducing the highest calculated EII by the top percent target, whilst the low bound level is determined by multiplying the highest calculated EII by the low bounded percent target. Any process above the maximum bound in the summary radar chart can be described as a critical process requiring Kaizen activities to reduce the overall EII value. Conditional formatting has been used to set the displayed summary process EII to red to reflect a poor EII performance if above the maximum bound. Processes that are between the bounds are ones that do not require immediate attention but have the potential to have a large EII over the next few EVSM iterations and are set to display yellow. Finally, processes below the minimum threshold are set to a green showing that they will most likely not require intervention.

The second use of the radar chart is to display a breakdown of each processes performance with respect to the chosen EIF. The first step is to determine the total sum of the total system EIFs Each process radar chart is then created by determining the percent contribution of that processes EIF to the total system EIF. The practitioner can easily compare and identify which environmental factor of what particular process requires Kaizen implementation (as shown in Figure 8). The final aspect of the index incorporation is the inclusion and transfer of the summary EII data onto the standard VSM templates creating the final EVSM product. An example representative EVSM is shown in Figure 7. The figure shows a representative standard value stream (yellow data boxes), standard "time" domain Kaizen, and associated lead time ladder. Below the lead time ladder is the main contribution of this paper, the inclusion of an integrated environmental impact ladder and associated environmental Kaizen linking key lean VSM use with environmental considerations.

5.5. Application to Industrial Case Study. The industrial case study under examination is an organisation that remanufactures aviation turbines. Quality of work is of utmost importance, due to the safety and reliability considerations. In addition, a rapid turnaround of the product is important for the client's utilisation of expensive airframes. The minimisation of environmental waste is important for both the client (the aviation industry is sensitive to carbon footprint) and the remanufacturer (toxicity of plating processes in particular). The environmental VSM approach was applied in this environment and results follow.

First, the firm identified the EIF to which it was sensitive (see Section 5.1). These were carbon footprint, perceived 


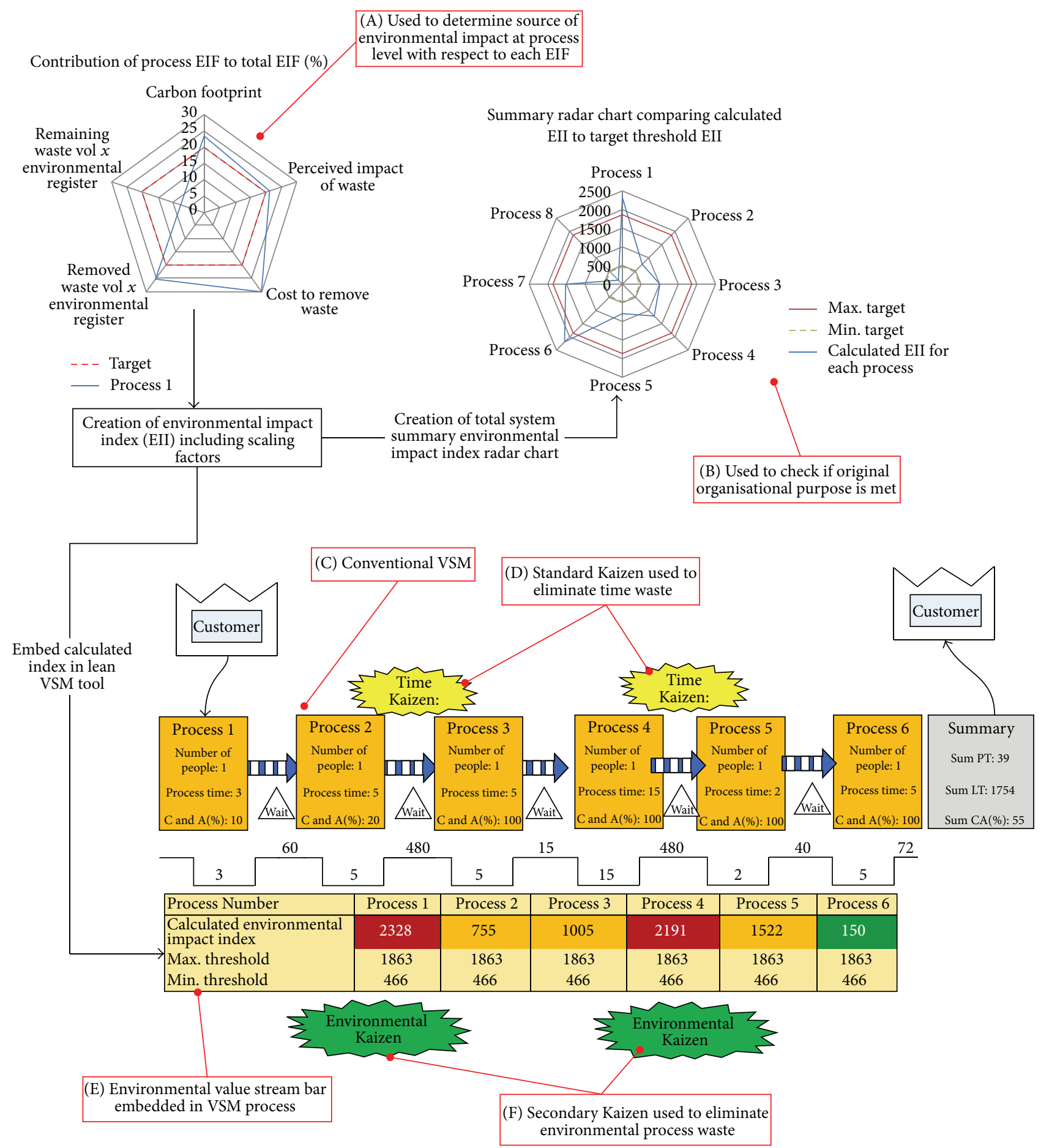

FIGURE 7: Illustration displaying the method in which final EII is aggregated from EIF data ((A) and (B)), the conventional time value stream $((C)$ and $(D))$, how the EII are incorporated into VSM (E), and the resulting environmental Kaizen created (F).

impact of waste, cost of cleanup/remediation, removed waste volume (weighted according to Site Environmental Risk Register), and remaining waste volume (likewise weighted). This was made for a total of five impacts (the methodology accepts any number). The environmental impacts were then assessed as part of a real VSM development.
5.5.1. Current State Environmental VSM. The EVSM method described was applied to a production process value stream within the industrial setting. A typical process stream might consist of between seven and a hundred activities depending on the level of detail required for analysis. The chosen value stream consisted of nine process stages that contained a large 


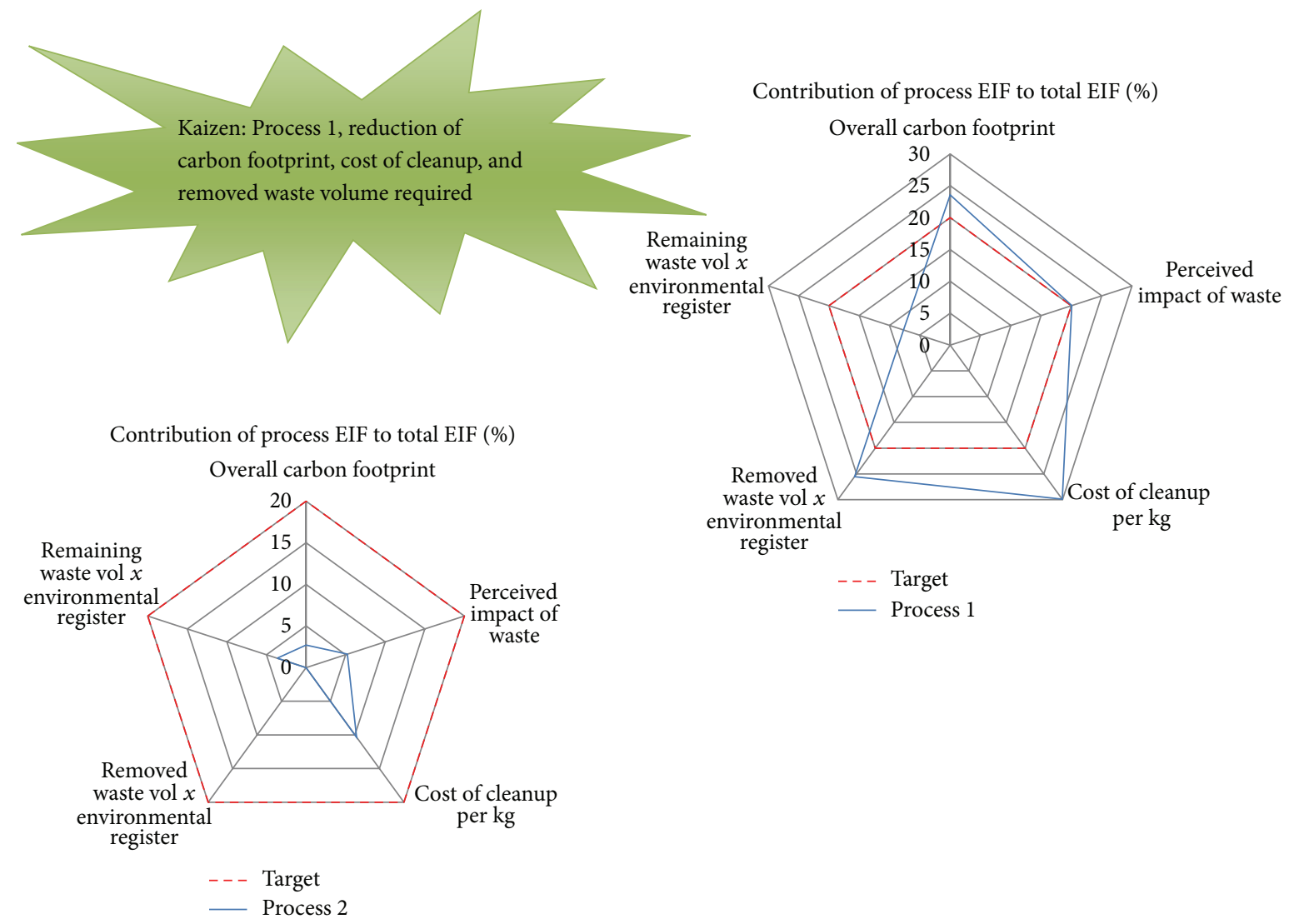

FIGURE 8: Representative sample and comparison of two process EIF radar charts and example of Kaizen for high environmental impact Process.

variety of environmental waste impacts. The method was applied to the VSM chosen and the EVSM created as shown in Figure 9.

5.5.2. Environmental Kaizen. This process resulted in a key Kaizen being created, for the high environmental impact Process 6, shown in Figure 10.

There is always a balance between economic and environmental goals during the continuous improvement process, and for this reason it is useful to have managerial representation in the Kaizen event. In principle the target future environmental waste levels can be included in the future state map.

5.6. User Survey of Applicability. To validate the effectiveness of the created EVSM method and associated index, a survey was conducted of industry participants. The questions relevant to the present study are as follows.

(i) Question 1: to what extent is it important to measure environmental waste impacts?

(ii) Question 3: to what extent does the practitioner feel the tool was successful in promoting new thinking and continuous improvement?

(iii) Question 4: to what extent does the practitioner feel the method was effective at identifying environmental waste impacts?
Responses are shown in Figure 11.

These results show that practitioners understood the importance of measuring the impact of environmental waste (Q1). They felt the tool was successful in promoting new thinking and continuous improvement (Q3) and effective at identifying environmental waste (Q4). The practitioners also felt the tool helped sensitise the user to the environmental impact of processes as well as show actual process level data attributing overall site wide data to source of environmental waste impact. We therefore conclude that the tool was successful in achieving the primary purpose.

\section{Discussion}

6.1. Outcomes: What Has Been Achieved? This work has made several contributions to the body of knowledge regarding environmental Value Stream Mapping and lean manufacturing principles. The first contribution is the creation of a method to integrate environmental and lean methods. Specifically we have shown integration from the generic environmental standard ISO 14001 through an organisational environmental risk register, onwards to integration within the VSM process, and thus finally permitting the established lean improvement process (e.g., kaizen) to be focussed at specific environmental improvement actions. Thus we have found a way to take the abstract concepts of environmental waste and make them concrete. Specifically, we have developed a 


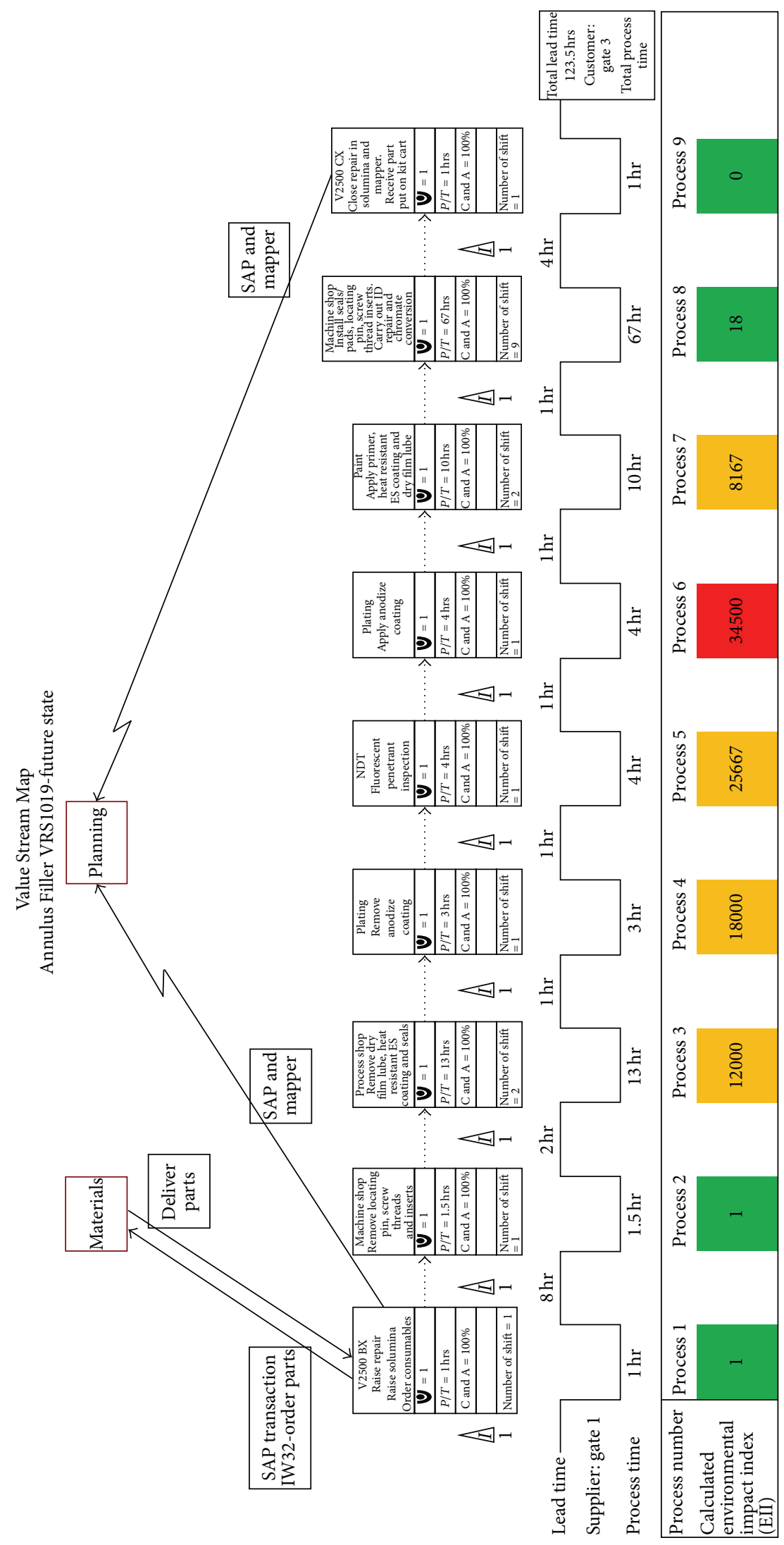

FIGURE 9: Implemented Environmental Impact Index incorporated with VSM for the chosen industrial value stream. 


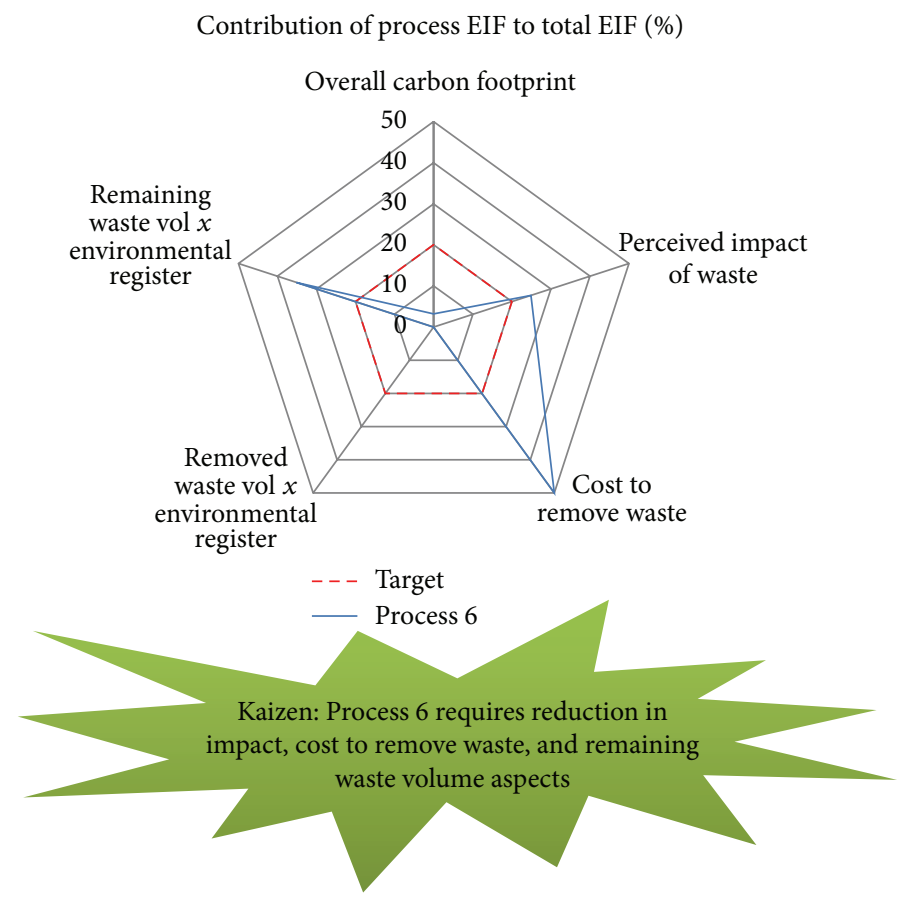

Figure 10: Process level radar chart providing key output: environmental Kaizen created in response to high impact process and waste identification through use of applied method and radar charts (Process 6-Plating: apply anodize coating).

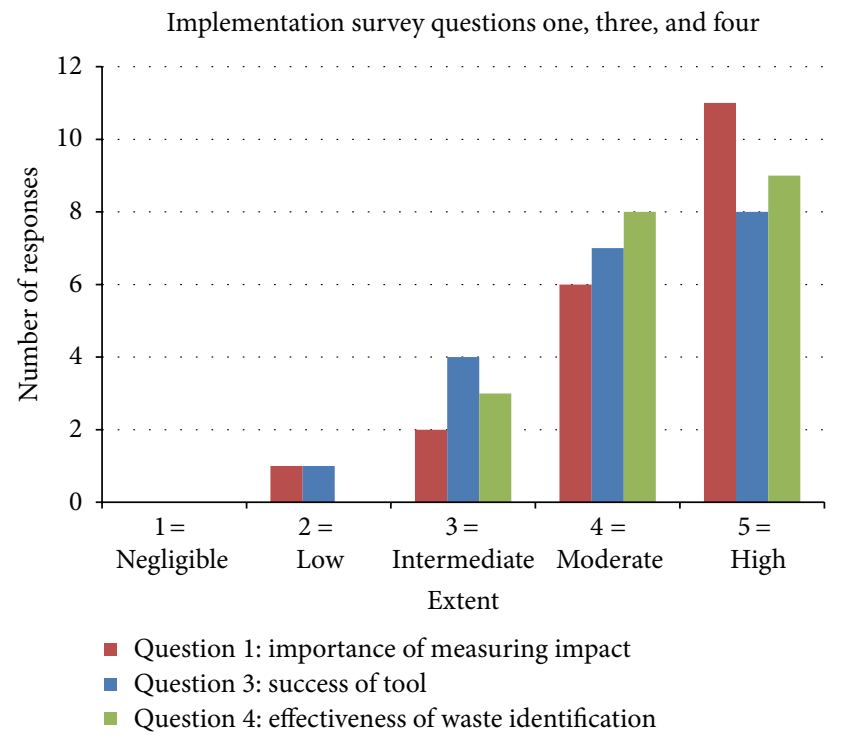

FIGURE 11: Results of survey questions one, three and four. These results demonstrate effectiveness of the method.

method to operationalise environmental waste within the VSM lean method. This methodology is shown in Figure 12.

A second contribution is the development of an $n$th dimension environmental factor methodology to create a customised environmental waste index for a particular industry. While the index created for this specific case used carbon footprint, perceived impact, cost to remediate, and waste volumes (removed and residual), the method is capable of being generalised to use different and any number of factors.
A third advancement is that we developed a way to use ambiguous user estimates of the quantity of each type of waste. This is important because it provides a basis for estimating values that are imprecise and otherwise difficult for operators to commit to a single deterministic value. Thus the method is capable of identifying areas for improvement (which is the overall purpose) despite ambiguous and imperfect information. To achieve this we used the PERT beta distribution, which already has acceptance in the project-management field.

A fourth contribution is the design of a way to represent the multidimensional environmental wastes that are relevant to diverse industry situations. Specifically we have used radar charts to help attribute process level environmental impact to overall system data and attribute environmental impacts to the source of the problem. This concept also allows the practitioner to drill down or up from a process to an overall system level (or vice versa) for the required information.

6.2. Implications for Practitioners. Industry practitioners at the production level now have a method to identify specific improvement activities (e.g., Kaizen) for environmental waste consistent with the organisational priorities. Thus environmental waste can be considered alongside other forms of waste during the VSM process (see Figure 12). To implement this, production engineers and supervisors would thus apply the environmental waste considerations as part of VSM (see action 2.2 in Figure 12). Then Kaizen solutions are developed Kaizen in the normal manner. Optionally, they can also report back to senior management against objectives for environmental waste and can do so at the level of whole value streams or individual processes. 


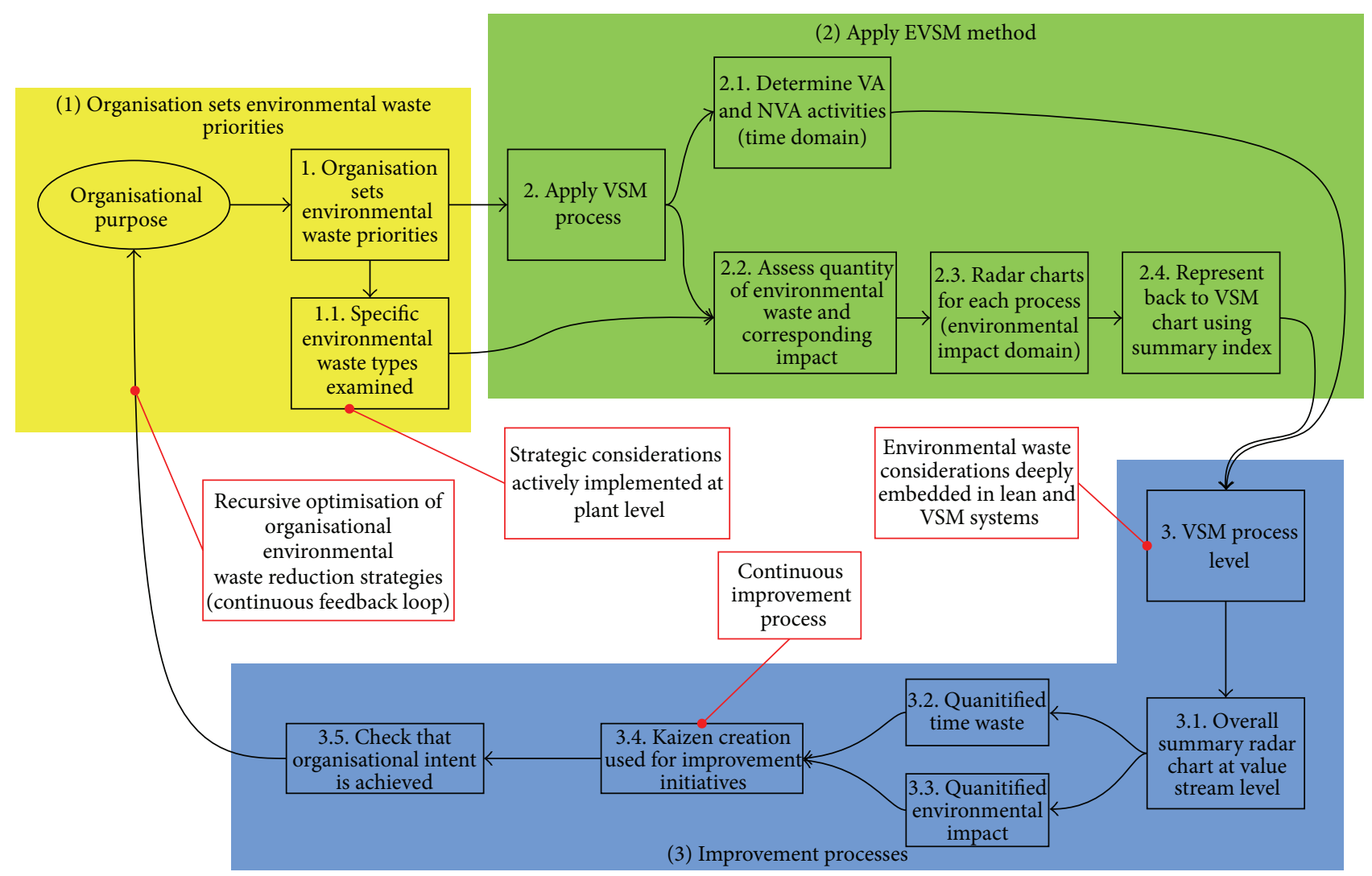

FIGURE 12: Summary of the overall method as implemented in industry.

Complementary to that, senior management now have a method to take the external environmental standard ISO 14001 and develop a customised construct for environmental waste for their particular organisation. They can then align the production processes, particularly the priorities going into the continuous improvement processes, to achieve those organisational objectives. Thus the method provides a strategic tool for firms that seek to improve their environmental position as summarised in Figure 12. A further implication from a management perspective is that the method has been developed with implementation and change management in mind. It has been specifically designed to be easy to implement and to fit in with existing organisational cultures. It achieves this by being complementary to the established practices of lean and VSM in particular: it takes advantage of practices and ideas with which the organisation is already familiar.

To implement this method, executives and production managers would decide on which environmental wastes to include and set the priorities for each (see activity 1 in Figure 12). Production staff would then implement this alongside the usual VSM processes. One of the quality staff would need training to use the method, but widespread training of other staff is not necessary (providing they already know how to implement VSM). Executives can then request summary information on overall environmental waste burden and efficacy of improvement measures. They can then use this information to further refine the strategic approach to manage the environmental waste.
There are many other industries that use lean principles, such as service organisations and project management. The method derived here is generic and not limited to manufacturing and therefore has potential applicability to these other areas. All organisations, including service firms, can identify waste priorities, assess their waste impacts, and implement Kaizen improvements. The concept of time is particularly relevant to service industries, so Value Stream Mapping is a particularly relevant lean tool in these situations.

6.3. Limitations and Opportunities for Further Research. During the implementation of the described method, several bottlenecks in usability of the system were discovered. The most notable bottleneck was the calculation component when determining the carbon footprint for each stage of the EVSM. This was remedied through the inclusion of an excel spreadsheet that determined carbon footprint for any process. The application of the method was also limited by the level of understanding of the practitioner with respect to environmental impacts and actual process level data instead of overall site level data.

An obvious limitation is that although we have integrated environmental waste with lean manufacturing practices, the integration is only for value stream mapping. There are many other lean methods, and not all organisations use VSM. Where time is the main driver of cost or quality, then VSM is appropriate, but this is not relevant to the production economics of all organisations. At present the integration has only been demonstrated for the manufacturing industry. 
Consequently there are opportunities for future research to extend and adapt the method to other situations.

\section{Conclusion}

This work develops a method to integrate evaluation of environmental impacts and lean methods. The method has been developed and tested in a manufacturing setting, and is able to represent a variety of environmental wastes within the Value Stream Mapping (VSM) method. Specifically it integrates from generic environmental standard ISO 14001, through organisational environmental risk register, onwards to integration within the VSM process, and thus finally permitting the established lean improvement process (e.g., Kaizen) to be focussed at specific environmental improvement actions. The deployment used carbon footprint, perceived impact, cost to remediate, and waste volumes (removed and residual), but the method is capable of being generalised to $n$th dimension environmental factors. It is thereby able to represent a customised environmental waste index for a particular industry. Ambiguous user estimates of waste quantities are accommodated through the PERT beta distribution. Several ways to represent the multi-dimensional environmental wastes were explored via industry focus group and the preferred representation designed to completion. The resulting method can be used by production staff and represents environmental impacts at the level of the individual process and aggregated to the whole value stream. The method may also be used by executives to align organisational practices with strategic objectives for waste reduction.

\section{Appendix}

\section{A. Implementation of VSM}

A VSM requires five steps that can then be applied to information, material, or process flow. A brief summary of the five steps is provided $[3,13]$.

A.1. Identify Target Product, Family, or Service. This stage requires the translation of customer requirements into process requirements. The customer base can be both external and internal and is described as those who accept, evaluate, install/inspect, own and use products or services.

A.2. Map Current State. Creating a current state VSM requires a team of people (who both manage and support various parts of the value stream) and who have been closely associated or involved with the process or information flow. Once the critical value stream has been chosen, every task or component is noted in the order that it is required to complete the service or product, starting at the shipping process and working backwards in the value stream to the raw materials or suppliers, while collecting information at each stage [10,15].

A.3. Asses Current VSM in terms of Creating a Better Flow by Eliminating Waste. Once the current state map has been completed, an assessment should be carried out to determine which processes add value. This step requires the identification of all value-added (VA) and nonvalue -dded (NVA) activities, as well as necessary but nonvalue-adding (NNVA). A common exercise used during this operation is the lean implementation tool called a "Kaizen burst" in which areas that represent large amounts of NV-added time (lead time) are targeted and reduced or eliminated. In this circumstance, a Kaizen event is one in which a process is critically reviewed to determine areas which could be improved.

A.4. Draw Future State VSM. Once the target waste (Kaizen initiatives) areas are identified, an ideal future state map (FSM) should be determined. This map should represent how the value stream will look after the identified waste has been eliminated and all Kaizen implemented. The FSM should be indicative of a situation in which all the individual processes produce only what its customer/process needs (or as close as possible) and only when required.

A.5. Work toward the Future State Condition. The final stage in VSM analysis is the creation and implementation of a work plan to accomplish the waste reduction goals identified whilst determining the FSM. The implementation plan describes how the goals set whilst creating the FSM are going to be achieved. Waste identification is a crucial element of any VSM as it is indicative of the Kaizen events held to reduce NVA activities. Some common reasons for waste within an information or manufacturing system are as follows [34]:

(i) push rather than pull based specifications and requirements,

(ii) nonoptimal use of human resource (e.g., using the wrong staff to do the wrong job such as a manager level or high engineering level staff doing NVA or NNVA work),

(iii) lack of detail, lack of organisation in planning, and lack of leadership and management,

(iv) use of obsolete two-dimensional drawings instead of single point release database with three-dimensional data.

\section{Acknowledgment}

The authors acknowledge the support of the Christchurch Engine Centre, New Zealand, particularly Tim Coslett for hosting and supporting this project.

\section{References}

[1] W. E. Deming, Out of the Crisis, MIT Press, Cambridge, Mass, USA, 1986

[2] J. Womack, D. Jones, and D. Roos, The Machine that Changed the World: The Story of Lean Production, Harper Perennial, 1991.

[3] J. P. Womack and D. T. Jones, Lean Thinking: Banish Waste and Create Wealth in your Corporation, Simon and Schuster, London, UK, 1996.

[4] F. A. Abdulmalek and J. Rajgopal, "Analyzing the benefits of lean manufacturing and value stream mapping via simulation: 
a process sector case study," International Journal of Production Economics, vol. 107, no. 1, pp. 223-236, 2007.

[5] T. Melton, "The benefits of lean manufacturing: what lean thinking has to offer the process industries," Chemical Engineering Research and Design, vol. 83, no. 6, pp. 662-673, 2005.

[6] P. Hines, M. Holwe, and N. Rich, "Learning to evolve: a review of contemporary lean thinking," International Journal of Operations and Production Management, vol. 24, no. 10, pp. 994-1011, 2004.

[7] B. J. Hicks, "Lean information management: understanding and eliminating waste," International Journal of Information Management, vol. 27, pp. 233-249, 2007.

[8] P. Hines and N. Rich, "The seven value stream mapping tools," International Journal of Operations and Production Management, vol. 17, no. 1, pp. 46-64, 1997.

[9] I. Serrano Lasa, R. D. Castro, and C. O. Laburu, "Extent of the use of Lean concepts proposed for a value stream mapping application," Production Planning and Control, vol. 20, no. 1, pp. 82-98, 2009.

[10] M. Braglia, G. Carmignani, and F. Zammori, A New Value Stream Mapping Approach for Complex Production Systems, Taylor and Francis, 2006.

[11] D. Tapping, T. Luyster, and T. Shuker, Value Stream Management, Productivity Press, A Division of Kraus Productivity Organization, 2002.

[12] Y. H. Lian and H. Van Landeghem, "Analysing the effects of Lean manufacturing using a value stream mapping-based simulation generator," International Journal of Production Research, vol. 45, no. 13, pp. 3037-3058, 2007.

[13] M. Rother and J. Shook, Eds., Learning to See: Value Stream Mapping to Add Value and Eliminate MUDA, The Lean Enterprise Institue, Brookline, Mass, USA, 1999.

[14] P. Kuhlang, T. Edtmayr, and W. Sihn, "Methodical approach to increase productivity and reduce lead time in assembly and production-logistic processes," CIRP Journal of Manufacturing Science and Technology, vol. 4, no. 1, pp. 24-32, 2011.

[15] D. Seth and V. Gupta, "Application of value stream mapping for lean operations and cycle time reduction: an Indian case study," Production Planning and Control, vol. 16, no. 1, pp. 44-59, 2005.

[16] B. Singh, S. K. Garg, and S. K. Sharma, "Value stream mapping: literature review and implications for Indian industry," International Journal of Advanced Manufacturing Technology, vol. 53, pp. 799-809, 2011.

[17] Y. Monden, Toyota Production System: An Integrated Approach to Just-in-Time, Industrial Engineering and Management Press, Norcross, Ga, USA, 1993.

[18] A. Gurumurthy and R. Kodali, "Design of lean manufacturing systems using value stream mapping with simulation A case study," Journal of Manufacturing Technology Management, vol. 22, no. 4, pp. 444-473, 2011.

[19] S. Irani, "Value Network Mapping (VNM): visualization and analysis of multiple interacting value streams in jobshops," in Proceedings of the 4th Annual Lean Management Solutions Conference, Institute of Industrial Engineers, Los Angeles, Calif, USA, September 2004.

[20] EPA, "EPA, The lean and Environmental Toolkit," Contract EPW-04-23, 2011, http://www.epa.gov/lean/environment/toolkits/ index.htm.

[21] ISO:14031, "Environmental Managment-Environmental Performance Evalutation Guidelines," ISO 14031:2000, International Organization for Standards, Geneva, Switzerland, 2000.
[22] W. McDonough and M. Braungart, Cradle to Cradle: Remaking the Way we Make Things, North Point Press, 2002.

[23] C. Jasch, "The use of Environmental Management Accounting (EMA) for identifying environmental costs," Journal of Cleaner Production, vol. 11, no. 6, pp. 667-676, 2003.

[24] A. A. King and M. J. Lenox, "Lean and green? An empirical examination of the relationship between lean production and environmental performance," Production and Operations Management, vol. 10, no. 3, pp. 244-256, 2001.

[25] S. Hart and G. Ahuja, "A natural resourced based view of the firm," Academy of Management Review, vol. 20, no. 4, pp. 9851014, 1995.

[26] A. Brinkley, M. Karlsson, J. R. Kirby, and D. Pitts, "Systematic environmental assessment: a decision methodology for strategic environmental issues," in Proceedings of the IEEE International Symposium on Electronics and the Environment (ISEE '00), pp. 178-183, San Francisco, Calif, USA, October 2000.

[27] S. Salhofer, G. Wassermann, and E. Binner, "Strategic environmental assessment as an approach to assess waste management systems. Experiences from an Austrian case study," Environmental Modelling and Software, vol. 22, no. 5, pp. 610-618, 2007.

[28] I. Hokerby, "Environmentally compatible product and process development," in Proceedings of the NAE Workshop on Corporate Environmental Stewardship, 1993.

[29] D. J. Richards, "Environmentally conscious manufacturing," World Class Design to Manufacture, vol. 1, no. 3, pp. 15-22, 1994.

[30] T. Wiedmann and J. Minx, "A defintion of, 'carbon footprint," in Ecological Economics Reserach Trends, C. C. Pertsova, Ed., chapter 1, Nova Science Publishers, New York, NY, USA, 2008.

[31] GRI, G3 Stustainability Reporting Guidelines, Global Reporting Initiative, Amsterdam, The Netherlands, 2006.

[32] A. S. Patil, "Incorporating environmetnal index as waste into value stream mapping," in Mechanical Eningeering, Masters of Science, Major of Industrical Engineering, p. 93, Wichita State University, Wichita, Kan, USA, 2002.

[33] A. S. Torres and A. M. Gati, "Environmental value stream mapping (EVSM) as sustainability management tool," in Proceedings of the Portland International Conference on Management of Engineering and Technology (PICMET '09), pp. 1689-1698, Portland, Ore, USA, August 2009.

[34] B. W. Oppenheim, "Lean product development flow," Systems Engineering, vol. 7, pp. 352-376, 2004. 

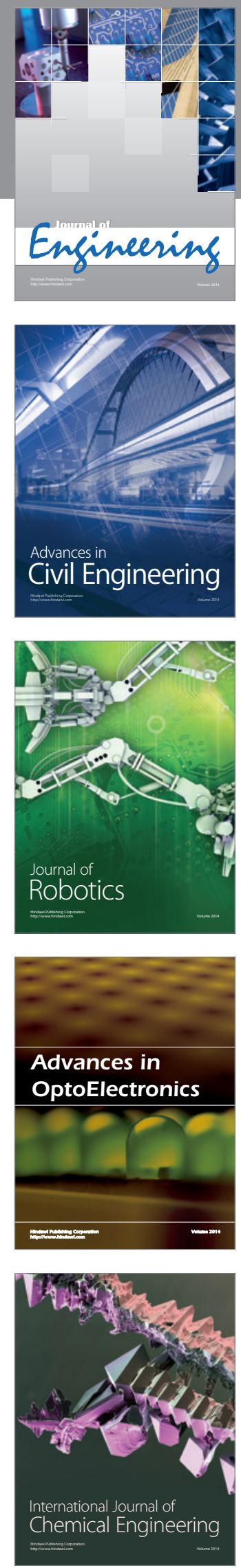

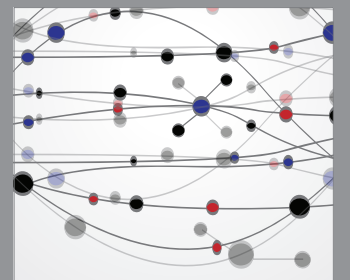

The Scientific World Journal
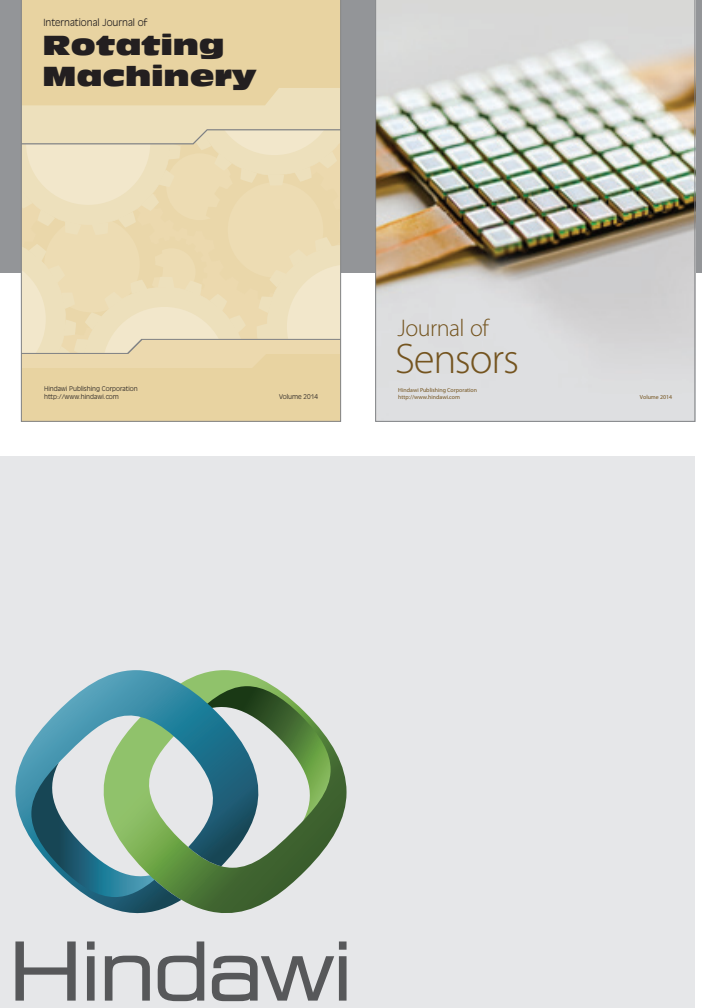

Submit your manuscripts at http://www.hindawi.com
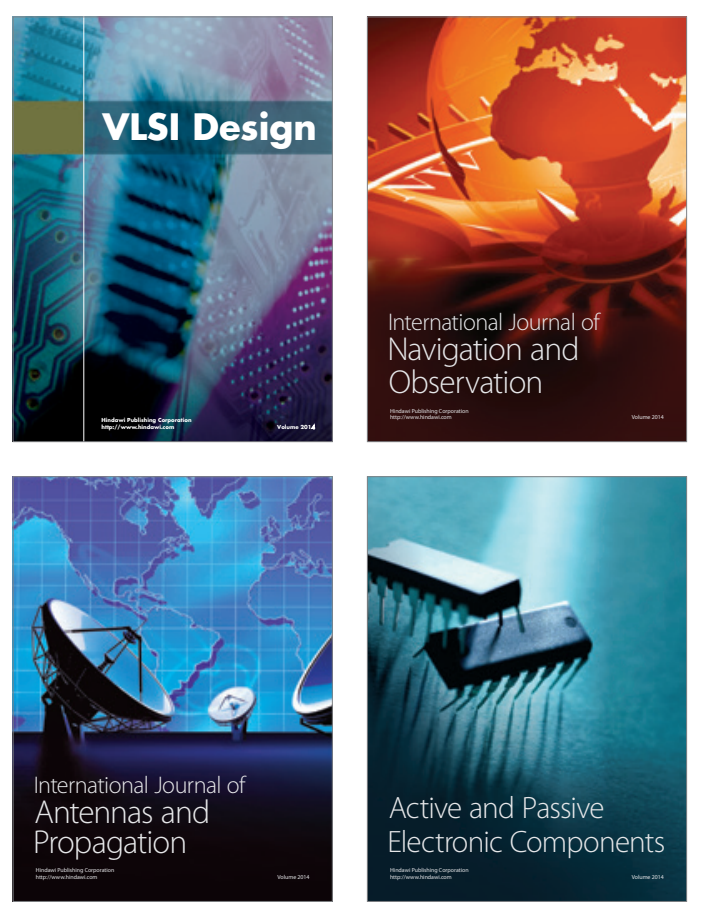
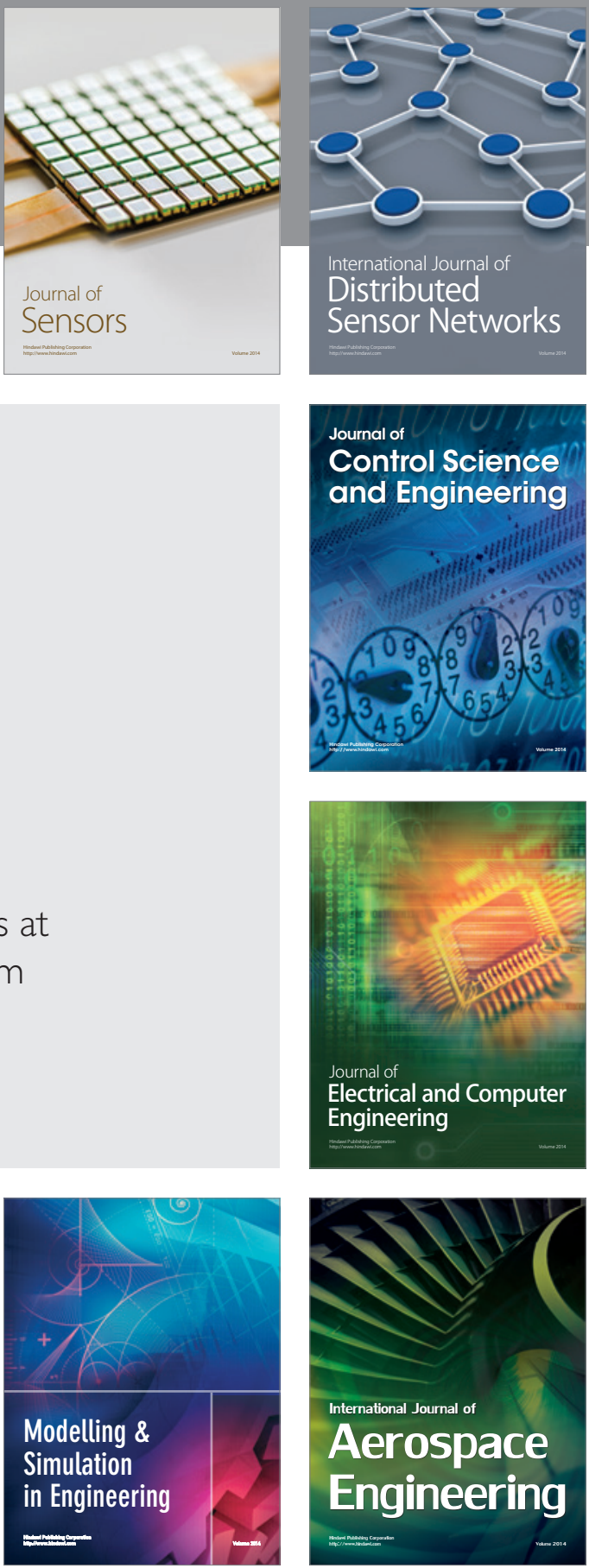

Journal of

Control Science

and Engineering
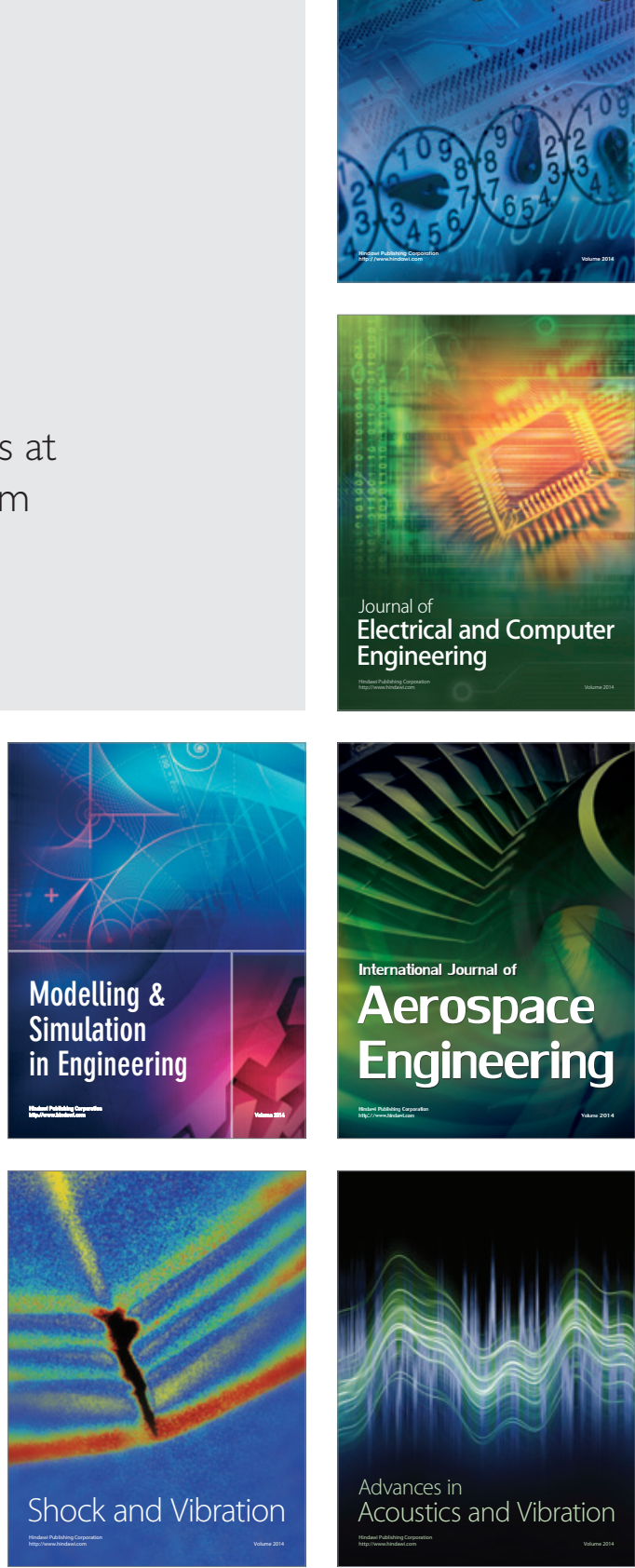